\title{
Chandra Studies of Supernova Remnants and Pulsars
}

\author{
Patrick Slane \\ Harvard-Smithsonian Center for Astrophysics, 60 Garden Street, \\ Cambridge, MA 02138, USA
}

\author{
John P. Hughes, Cara E. Rakowski \\ Department of Physics and Astronomy, Rutgers University, 136 \\ Frelinghuysen Road, Piscataway, NJ 08854-8019, USA \\ David N. Burrows, John A. Nousek, Gordon P. Garmire \\ Department of Astronomy and Astrophysics, 525 Davey Laboratory, \\ Pennsylvania State University, University Park, PA 16802, USA
}

\author{
Bryan M. Gaensler \\ Center for Space Research, Massachusetts Institute of Technology, \\ Cambridge, MA 02139, USA.
}

\begin{abstract}
With sub-arcsecond angular resolution accompanied by fast time resolution and spatially resolved spectral capabilities, the Chandra X-ray Observatory provides a unique capability for the study of supernova remnants (SNRs) and pulsars. Though in its relative infancy, Chandra has already returned stunning images of SNRs which reveal the distribution of ejecta synthesized in the stellar explosions, the distinct properties of the forward and reverse shocks, and the presence of faint shells surrounding compact remnants. Pulsar observations have uncovered jet features as well as small-scaled structures in synchrotron nebulae. In this brief review we discuss results from early Chandra studies of pulsars and SNRs.
\end{abstract}

\section{Introduction}

The X-ray emission from SNRs provides immense detail regarding the temperature, composition, distribution, and ionization state of material synthesized and ejected in SN explosions, as well as that for swept-up material from the circumstellar and interstellar medium (CSM and ISM, respectively). Significant advances in our understanding of SNR structure and dynamics have been made through X-ray observations carried out with imaging observatories such as Einstein, ROSAT, and ASCA. Angular resolution on order of 5-10 arcsec with ROSAT and Einstein provided evidence of small-scale structure in SNRs, but without spectral information. Moderate spectral resolution and a broader energy bandpass allowed ASCA to probe elemental abundances and ionization states, but only over angular regions of several arcmin. All of these capabili- 

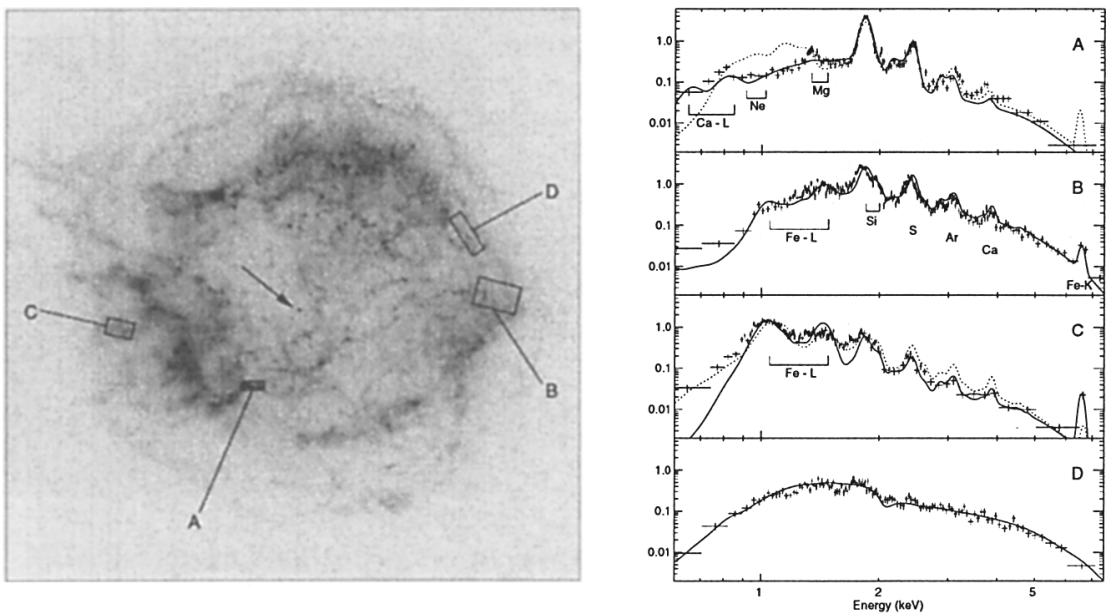

Figure 1. ACIS-S image of Cas A. Regions used for spectral extraction are shown; an arrow marks the central point source. Spectra from indicated regions are shown at right. (From Hughes et al. 2000)

ties, with much higher angular resolution, are provided by the Chandra X-ray Observatory, and the impact on studies of SNRs is already being felt. In this review of early Chandra studies of SNRs and pulsars, we attempt to summarize a broad range of scientific issues, but concentrate on those aspects for which the high spatial resolution provided by Chandra plays a crucial role.

\section{Supernova Ejecta: Composition and Distribution}

The X-ray spectra of very young SNRs is typically dominated by emission from the ejecta. Large-scale mixing of these ejecta is of particular importance for constraining explosion models in which neutrino heating and convection in the neutrinosphere and shock front region may produce velocity, density, and temperature variations in the initial postshock region (e.g. Janka \& Mühler 1996). These variations may ultimately result in large-scale mixing such as that inferred for SN 1987A, where newly synthesized ${ }^{56} \mathrm{Ni}$ was apparently transported out to the hydrogen envelope, although difficulties in modeling such an overturn of the ejecta remain (e.g. Kifonidis et al. 2000).

With high angular resolution, Chandra allows the spectral investigation of discrete clumps of emission in young Galactic SNRs. Such an investigation has been carried out with the "first light" observation of Cas A by Hughes et al. (2000). The ACIS-S image of Cas A is shown in Figure 1, with spectra of indicated regions shown at the right. The dotted curves for regions $\mathrm{A}$ and $\mathrm{C}$, and the solid curve for B, represent models in which abundances are fixed to values appropriate for incomplete Si burning, which occurs just outside the stellar core (Thielemann, Nomoto, \& Hashimoto 1996). The material from region B is welldescribed by the model, indicating that the material originated near the core. Region A shows a deficit of $\mathrm{Fe}$, indicating that it was formed further from the core; the solid curve shows abundances appropriate for O-burning, which better 

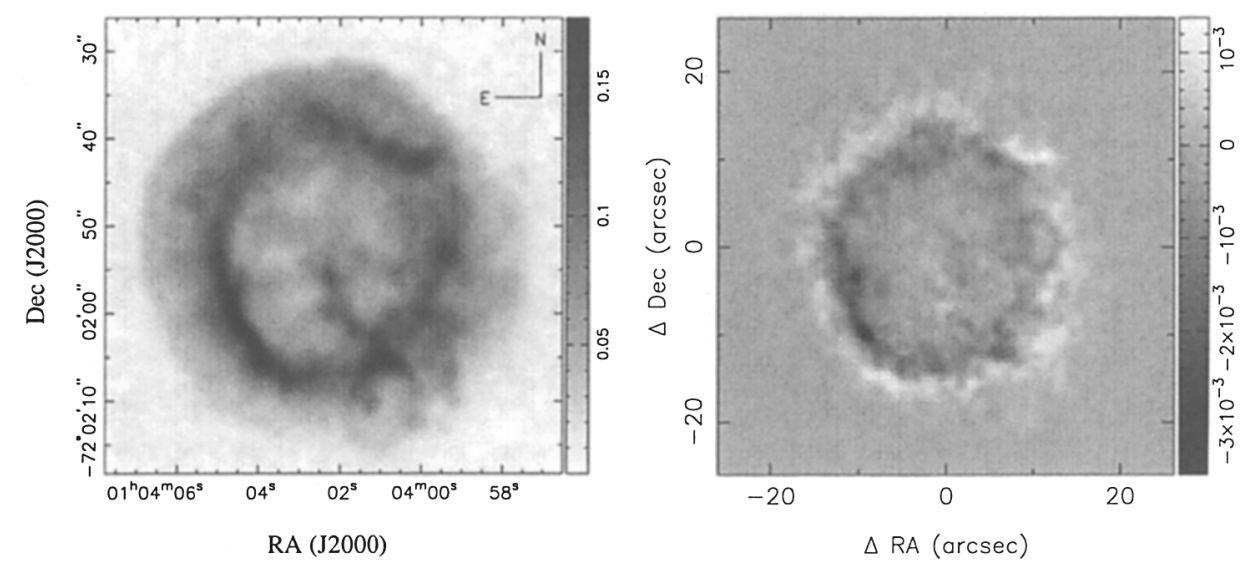

Figure 2. Left: ACIS-S image of 1E0102.2-7219. Right: Difference image of $\mathrm{O}$ VIII (white) and O VII (black) emission from 1E0102.2-7219. (Images courtesy of T. Gaetz.)

describes the data, and indicates that this material was formed outside the Siburning zone. Region $\mathrm{C}$, however, shows even more Fe-K than expected from incomplete Si-burning, indicating that it originated even closer to the core.

The radial distribution of regions $\mathrm{A}, \mathrm{B}$, and $\mathrm{C}$ suggest an overturn of the ejecta; material formed closest to the stellar core $(\mathrm{C})$ is found further from the remnant center than that formed outside the core $(\mathrm{A})$. This indication is strengthened by the analysis of Hwang, Holt, \& Petre (2000) who, using a much deeper observation, produce equivalent-width images showing that the $\mathrm{Fe}$ layers are located predominantly outside of the Si layers. They also show that the remaining ejecta distribution is similar to that of the fast-moving optical knots, but that the hard continuum morphology differs from that of the ejecta.

\section{SNR Shock Structure and NEI Effects}

As the blast wave sweeps up ambient material, the increase in pressure drives a reverse shock into the ejecta, resulting in the formation of two distinct X-ray emitting zones - the forward shock-heated CSM/ISM component associated with the explosion blast wave, and the hot ejecta associated with the reverse shock. With high resolution X-ray observations, these components can be separated spatially, and the spectral properties of each can be measured. This is evident in Figure 2 which shows the Chandra ACIS image of 1E0102.2-7219 (Gaetz et al. 2000), an O-rich SNR in the SMC. The spectrum of the faint outer halo of emission reveals abundances consistent with interstellar material in the SMC (Hughes, Rakowski, \& Decourchelle 2000), while the bright ring-like feature in the interior region reveals large overabundances indicative of the SN ejecta.

When the SNR ejecta encounters the reverse shock, the temperature is increased and ionization occurs. However, because the density is low, the ionization state initially lags behind that expected for collisional equilibrium. Such 
nonequilibrium ionization (NEI) effects can result in radial variations of the ionization state, as the reverse shock progresses into the ejecta. This is demonstrated clearly in Figure 2 (right) which shows a difference image of the O VIII and O VII emission from 1E0102.2-7219 (Gaetz et al. 2000). The O VIII emission (white) resides outside of the O VII emission (black), indicating the time lag in ionizing up to O VIII as the reverse shock moves inward. This effect is shown more dramatically in images of 1E0102.2-7219 taken with the high energy transmission grating (HETG) on Chandra, which also show clear velocity structure in the Ne emission from the remnant shell, indicating that material is predominantly redshifted along the eastern limb, but both redshifted and blueshifted along the western limb (Flanagan et al. 2001).

\section{Nonthermal Emission from Shell-Type SNRs}

While the shock-heated ejecta and CSM/ISM components of shell-type SNRs produce line-dominated X-ray spectra, as described above, a growing number of remnants also reveal evidence of hard, nonthermal X-ray emission components, apparently associated with high energy electrons accelerated by the SNR shock. In three cases - SN 1006 (Koyama et al. 1995), G347.3-0.5 (Koyama et al. 1997; Slane et al. 1999), and G266.2-1.2 (Slane et al. 2001) - the nonthermal emission components completely dominate the thermal components, and the $\mathrm{X}$-ray spectra from the shells are featureless.

Particle acceleration by SNR shocks has, of course, been suggested for decades as a process by which cosmic rays are produced. Radio emission from SNRs provides ample evidence of electrons with $\mathrm{GeV}$ energies. However, it is the recent detections of nonthermal X-rays from shell-type SNRs that provide direct evidence of particles accelerated to energies as high as $10-100 \mathrm{TeV}$, approaching the knee in the cosmic ray spectrum. Models for nonlinear shock acceleration (e.g. Ellison, Berezhko, \& Baring 2000) predict a strong correlation between the radio, X-ray, and $\gamma$-ray flux that allow parameters of the acceleration process to be derived. It is thus of considerable interest to localize the regions of nonthermal X-ray emission, and to investigate the broad-band spectra (particularly in radio, X-rays, and $\gamma$-rays) from these regions. High spatial resolution in the X-ray band is particularly important in this regard, as is demonstrated in Figure 1. Here, the emission from a narrow filament in Cas A, identified as region " $\mathrm{D}$," is clearly represented by a featureless X-ray spectrum (Hughes et al. 2000). It would appear that an isolated site of efficient particle acceleration has been localized with this observation.

Further evidence of significant particle acceleration has been put forth in a study of 1E0102.2-7219 by Hughes, Rakowski, and Decourchelle (2000). Using Chandra data, these authors measure the SNR expansion rate through comparison with Einstein and ROSAT images. Assuming standard conditions for a nonradiative shock, the inferred expansion rate of $\sim 6000 \mathrm{~km} \mathrm{~s}^{-1}$ corresponds to a shock temperature of $2.5-45 \mathrm{keV}$, dependent upon the degree of collisionless heating. However, the spectrum of the outer shell yields a temperature of only $0.4-1 \mathrm{keV}-$ much lower than suggested by the expansion velocity. The conclusion is that a significant fraction of the shock energy has gone into particle acceleration and is thus unavailable for the heating of the electrons and ions. 


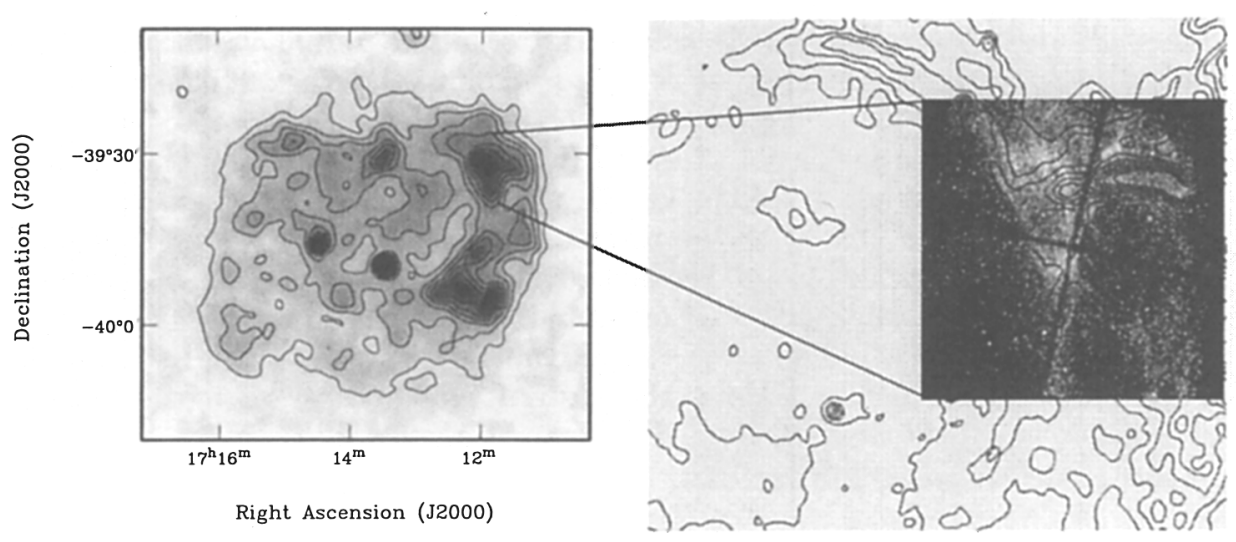

Figure 3. Left: ROSAT PSPC image of G347.3-0.5. Right: Chan$d r a$ image of northwest rim, with radio contours from ATCA.

In Figure 3 we present a preliminary Chandra image of the X-ray emission from the NW limb of G347.3-0.5, whose shell emission is nonthermal. Overlaid are radio contours from the Australia Telescope Compact Array (ATCA), showing good general correspondence between the components. This region of the SNR has also been detected as a source of $\sim \mathrm{TeV} \gamma$-rays (Muraishi et al. 2000). Preliminary modeling suggests a self-consistent picture in which the X-ray and radio flux is synchrotron radiation from the accelerated electrons, while the $\gamma$ ray flux represents inverse-Compton scattering of energetic electrons off of the microwave background (Ellison, Slane, \& Gaensler - in preparation).

\section{Pulsars and Synchrotron Nebulae}

Since the majority of $\mathrm{SNe}$ result from massive star collapse, and most such collapses are expected to form neutron stars (NSs), it is reasonable to expect that young NSs should be associated with most young SNRs. This is particularly true for cases in which properties of the SN events, or the SNRs themselves, point to a massive progenitor. The oxygen-rich SNRs, of which Cas A is a prime example, are firmly believed to be the product of such progenitors, yet until recently only one of seven such remnants was known to house a young NS (SNR 0540-6944 in the LMC).

Chandra HRC observations of SNR 0540-6944 (Gotthelf \& Wang 2000) reveal an extended synchrotron nebula which, through use of time gating on the $50 \mathrm{~ms}$ period of the associated pulsar PSR 0540-69, can be imaged separately from the pulsar itself. These observations reveal a roughly toroidal structure, which may represent the termination shock where the pulsar wind is confined within the surrounding nebula, along with faint evidence for a jet. This is strikingly reminiscent of the Crab Nebula X-ray structure, revealed in wonderful detail by Chandra observations (Weisskopf et al. 2000) wherein a clear toroidal structure is observed. A jet emanates from the pulsar along the symmetry axis of the torus, aligned with the position angle of the pulsar proper motion. An 


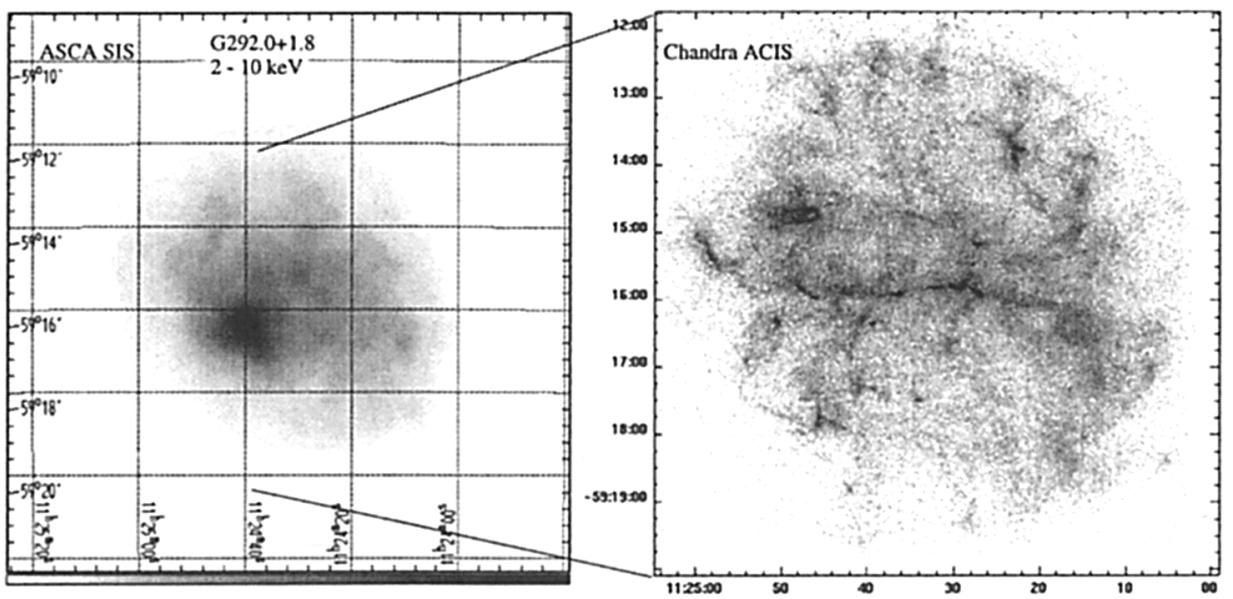

Figure 4. Left: ASCA SIS (2-10 keV) image of G292.0+1.8, showing enhanced emission possibly associated with a young NS. (From Torii, Tsunemi, \& Slane 1998.) Right: ACIS-S image of G292.0+1.8. A point source is detected at the position of the hard ASCA source.

inner ring structure with small-scale knots appears to coincide with the position of wisps observed in optical images. Remarkably, Chandra observations of the Vela pulsar reveal strikingly similar structure to that seen in the Crab. A toroidal structure within the synchrotron nebula is observed, along with a jet that is aligned with the pulsar proper motion.

Chandra observations of Cas A and G292.0+1.8 appear to show that SNR 0540-6944 is no long alone in the class of O-rich SNRs with associated (though not necessarily Crab-like) NSs. The Chandra image of Cas A (Figure 1) reveals the presence of a point-like source at the remnant center. The X-ray properties are unlike those of typical young pulsars, however. A power law model yields a photon index of $\sim 2.8-3.6$ (Chakrabarty et al. 2001), much steeper than that for a Crab-like pulsar. The luminosity $L_{x} \sim 10^{35} \mathrm{erg} \mathrm{s}^{-1}$ is also rather low when compared with other young pulsars. Alternatively, a blackbody model yields a temperature of $\sim 0.5 \mathrm{keV}$, but with a luminosity requiring an emitting area considerably smaller than the full surface of a typical NS, possibly implying emission from hot spots on the NS surface (Pavlov et al. 2000).

Evidence for a NS associated with G292.0+1.8 first came from ASCA observations. As seen in Figure 4 (left), the SIS data reveal a compact region of hard X-ray emission which Torii, Tsunemi, \& Slane (1998) have interpreted as originating from a pulsar-driven synchrotron nebula. Chandra ACIS observations of this remnant clearly reveal a point source of emission at the same location (Figure 4, right). Hard-band imaging reveals diffuse emission that is suggestive of a surrounding synchrotron nebula as well.

Although we infer the presence of an active, young NS with any X-ray synchrotron nebula, it is by no means the case that pulsars have been identified for each. G21.5-0.9, for example, is a well-defined plerion for which radio observations show no direct evidence of a compact central source. Chandra observations (Slane et al. 2000) reveal a compact source at the center of the nebula, but the 


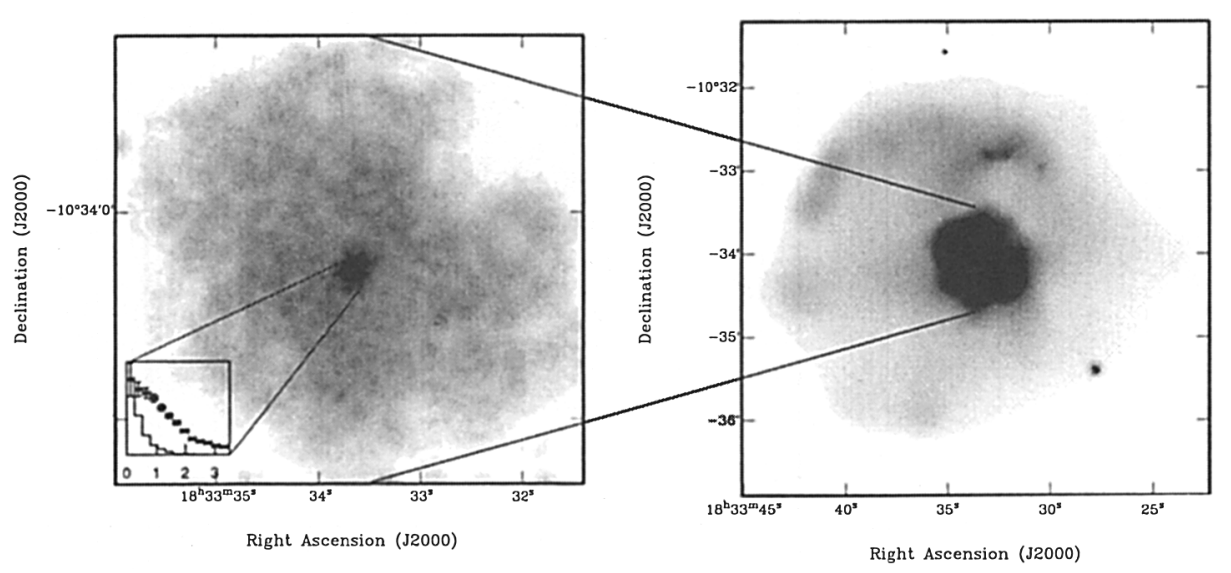

Figure 5. Left: HRC observation of G21.5-0.9. The inset compares the profile of the compact central emission (points) with that from a point source (histogram). Right: ACIS-S image showing the faint shell of emission surrounding the plerion. (From Slane et al. 2000)

high resolution images clearly demonstrate that the source is slightly extended (Figure 5). Using the radio spectrum to estimate the magnetic field strength of the nebula under the assumption of equipartition between the field and the particles, and requiring that this associated magnetic pressure act to confine a pulsar wind driven by the spin-down power, $\dot{E}$, of a central pulsar (using an empirical relationship between $\dot{E}$ and $L_{x}$ - see Seward \& Wang 1988) indicates that the extended size of the central emission is consistent with the radius of the stand-off shock where the wind is confined. As the particle wind traverses the standoff shock, the nebular structure changes from a particle-dominated to a magnetic-dominated flow (Kennel \& Coroniti 1984). The finite lifetime of the synchrotron emitting electrons results in a steepening of the spectrum with radius, an effect clearly observed in G21.5-0.9 (Slane et al. 2000).

At low surface brightness levels, the Chandra observations of G21.5-0.9 provide one additional piece of new information about this remnant - the presence of an extended halo of emission (Figure 5 - right). This may represent the blastwave component, with a very low ambient density resulting in weak emission. The spectrum of the halo is featureless, suggesting nonthermal shell emission such as that discussed in Section 4. Similar results have been reported based on observations with XMM-Newton (Warwick et al. 2001), although these authors interpret the halo as an extension of the bright synchrotron nebula. Deeper radio observations should help determine which (if either) scenario is correct.

\section{Conclusions}

High angular resolution X-ray observations offer a unique means by which to study the ejecta and shock structure of SNRs. With resolution approaching 
that of ground-based optical telescopes, Chandra provides the opportunity for significant advances in our ability to unfold the characteristics of remnants and their associated pulsars. Early observations have already broken new ground in this regard, including the identification of small-scale structures in pulsar nebulae, composition and ionization state variations in SN ejecta, and sites of particle acceleration by SNR shocks. Detailed comparisons with high resolution data from other wavelength bands promises to broaden our understanding of SNR dynamics and evolution, as well as to constrain and challenge theoretical modeling of these systems and their environments.

Acknowledgments. This work was supported in part by NASA through contract NAS8-39073 and grant NAG5-9106.

\section{References}

Chakrabarty, D., Pivovaroff, M. J., Hernquist, L. E., Heyl, J. S., \& Narayan, R. 2001, to appear in ApJ, Vol. 546

Ellison, D. C., Berezhko, El G., \& Baring, M. G. 2000, ApJ, 540, 292

Flanagan, K. A. 2001 - to appear in Proc. of 11th Annual Oct. Astrophysics Conference in MD, Young Supernova Remnants, ed. U. Hwang

Gaetz, T. J. et al. 2000, ApJ, 534, 47L

Gotthelf, E. V., \& Wang, D. Q. 2000, ApJ, 532, L117

Hughes, J. P., Rakowski, C. E., Burrows, D. N., \& Slane, P. O. 2000, ApJ, 528, L109

Hughes, J. P., Rakowski, C. E., \& Decourchelle, A. 2000, ApJ, 543L, 61

Hwang, U., Holt, S. S., \& Petre, R. 2000, ApJ, 537, L119

Janka, H.-T., Müller, E. 1996, A\&A, 306, 167

Kennel, C. F., Coroniti, F. V. 1984, ApJ, 283, 710

Kifonidis, K., Plewa, T., Janka, H.-Th., \& Müller, E. 2000, ApJ, 531, L123

Koyama, K. et al. 1995 Nature 378, 255

Koyama, K. et al. 1997, PASJ, 49, L7

Muraishi, H. et al. 2000, A\&A, 354, L57

Pavlov, G. G., Zavlin, V. E., Aschenbach, B., Trümper, J., \& Sanwal, D. 2000, ApJ, 531, L53

Seward, F. D., \& Wang, Z.-R. 1988, ApJ, 332, 199

Slane, P. et al. 1999, ApJ, 525, 357

Slane, P. et al. 2000, ApJ, 533, L29

Slane, P. et al. 2001, ApJ - accepted (see astro-ph/0010510)

Thielemann, F. K., Nomoto, K., \& Hashimoto, M. 1996, ApJ, 460, 408

Torii, K., Tsunemi, H., \& Slane, P. 1998, in IAU Symposium 188, The Hot Universe, ed. K. Koyama, S. Kitamoto, \& M. Itoh (Dordrecht: Kluwer Academic), 258

Warwick, R. S. et al. 2001, A\&A - accepted (see astro-ph/0011245)

Weisskopf, M. C. et al. 2000, ApJ, 536, L81 